\title{
Why Do Not All Chemotherapy Patients Lose Their Hair? Answering an Intriguing Question
}

\author{
Alfredo Rebora Marcella Guarrera \\ Department of Dermatology, University of Genoa, Genoa, Italy
}

Alopecia is one of the side effects of chemotherapy that patients fear most; to the point that, just for that reason, up to $14 \%$ of women refuse chemotherapy. In fact, chemotherapy drugs destroy cancer rapidly growing cells, but do not spare other rapidly growing cells, including hair keratinocytes. In temporary alopecia, full regrowth is, however, possible. Within $96 \mathrm{~h}$, DNA is repaired and hair bulb structures are restored [1], and within 3-6 months, new hairs are visible [2]. In $65 \%$ of patients, they acquire temporary different features, including curliness in $35 \%$ of cases [3]. Permanent or persistent alopecia (PA) is another possible outcome. High-dose busulfan and cyclophosphamide regimens, but breast cancer protocols as well, often including taxanes, are reported to cause it [4]. Almost constantly, all hairy areas are involved. The severest forms occur in up to $10 \%$ of cases and seem to be associated with regulatory variants in the ABCB1 gene [5].

The literature on the topic is remarkably vast and well documented but fails to answer an intriguing question: why do only some of the treated patients lose their hair, no matter the quality and dosage of the drug? The issue is quite complicated possibly because most of the researchers are not fully aware of the multifaceted aspects of trichology. Dermatologists as well often fail to deliver reliable data, as Tosti et al. [6] properly pointed out. According to Tallon et al. [7], for example, the histopathology of PA does not reveal any inflammatory sign, while scarring

karger@karger.com

(c) 2021 S. Karger AG, Basel

www.karger.com/sad

Karger" alopecias have been described [2] quite probably resulting from inflammation, and trichodynia [8], which may depend on peribulbar inflammation, has been reported in up to $87 \%$ of cases [9].

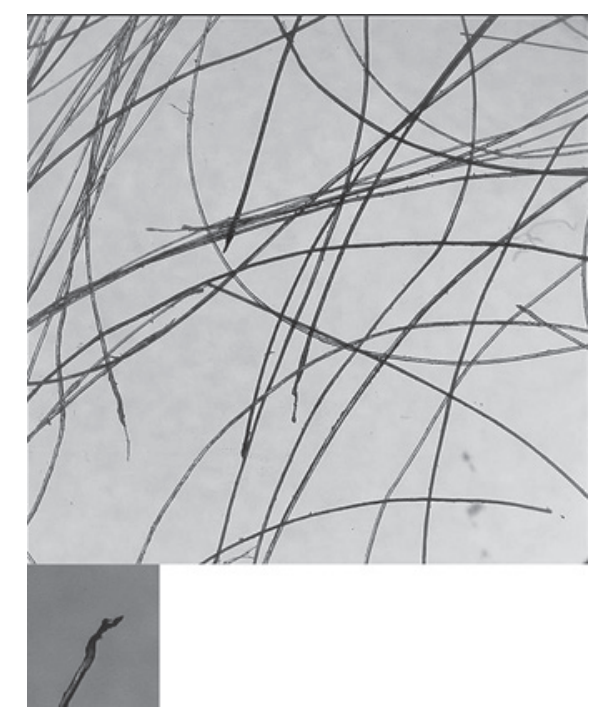

Fig. 1. AE of scalp hairs 5 days after the first infusion of docetaxel. Numerous dystrophic hairs are visible. In the small picture, a typical dystrophic root.
Correspondence to:

Alfredo Rebora, rebdermo@yahoo.it 
Fig. 2. a Right cheek of the same patient as in Fig. 1, 1 month after the first docetaxel infusion. Empty spaces are clearly visible. Black dots and white dots correspond to hairs that have been truncated at their origin (cadaverized hairs) as the result of AE. Many white hairs have grown up though and seem to be unaffected by dystrophy. b Left cheek, 55 days after the first docetaxel infusion and 3 days after the fifth infusion. Black and white dots are still present. Surviving white hairs are grown up but less than expected. Note the different magnification of the picture. AE, anagen effluvium.
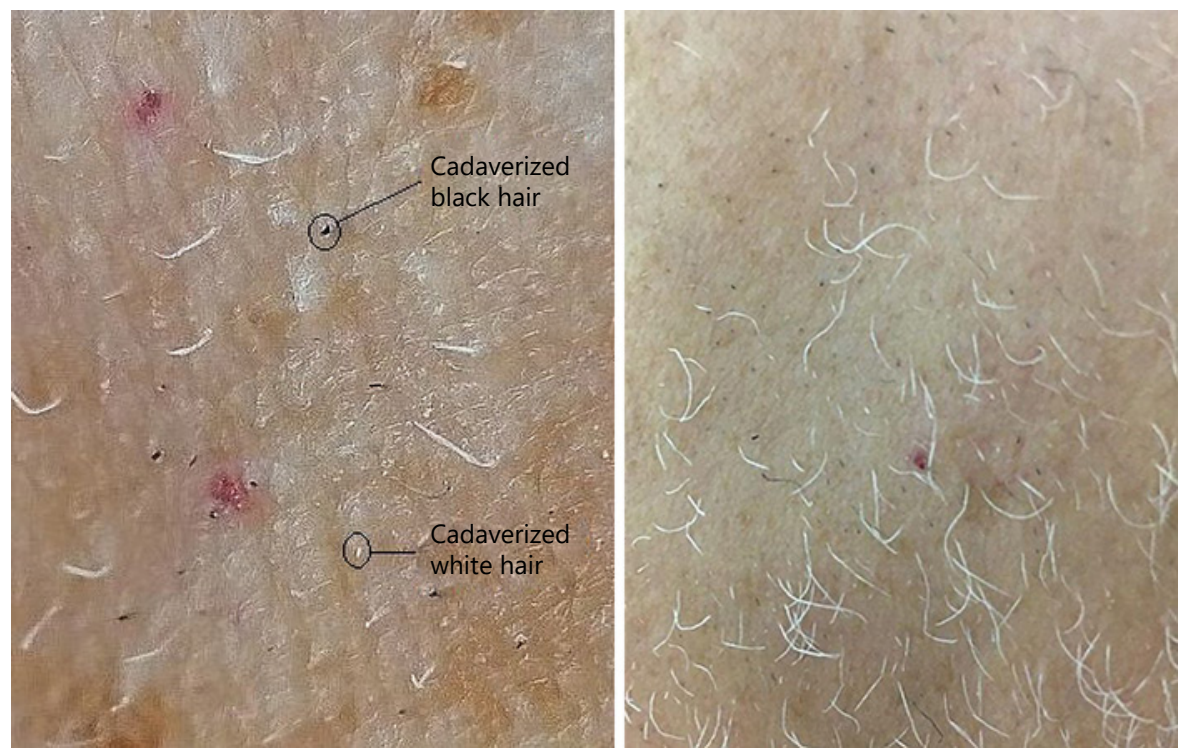

Indeed, apparently, irrespective of the quality and dose of the employed drugs, not all the treated patients complain of hair loss. In a series of Sengelov et al. [10], for example, the combination of docetaxel at $75 \mathrm{mg} / \mathrm{m}^{2}$ and cisplatin at $75 \mathrm{mg} / \mathrm{m}^{2}$ every 21 days caused alopecia in only $44 \%$ of patients. In another series, hairs were maintained in $31.3 \%$ of patients, regardless of the type of cancer and quality of the cytostatic drug [11]. In addition, seldom has mention been made of beard hairs [12] as if they were invulnerable. Other body hairs, such as eyebrows and pubic and axillary hairs, may be attacked as well.

The current understanding of the problem takes into main consideration the high mitotic rate of hair keratinocytes, which, in fact, "approach the maximum proliferative rate of keratinogenic epithelial cells" [13]. Hair keratinocytes are, just for that reason, particularly susceptible to any cytostatic insult. Stem cells are ordinarily spared, but in PA, they become the likely target of an undue antimitotic activity [2].

Oncologists, who are obviously concerned with this problem, acknowledge that "several factors may contribute to the severity of hair loss including drug, dose, and schedule" [14]. Purpose of this essay is to discuss some of those "several" unspecified factors that come into play. Three of them deserve to be considered here, focusing on the peculiar aspects of the target, the hair follicle, rather than on the drugs: the different mitotic rates of hair follicles in the various hairy areas, the effect of age on cell division rates, and the prevalence of androgenetic alopecia (AGA).

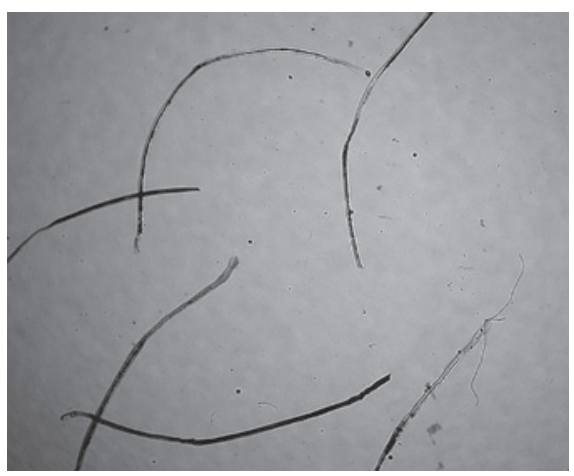

Fig. 3. Some of the white beard hairs in Fig. 1, which have however grown up, have been plucked and examined under a microscope. They were dystrophic hairs, except one possibly telogen hair.

Indeed, the drug interrupts the anagen phase of the hair cycle [15], arresting the mitoses of keratinocytes in the hair matrix. The expected consequence is a profuse loss of dystrophic hairs (anagen effluvium [AE]) (Fig. 1). However, as it often occurs in biology, things are not so simple. When an antimitotic insult affects an ordinary cell, the final result depends on 2 factors: the strength of the injury and/or its duration. Different from other epithelial tissues, though, the hair follicle is a dynamic target, going through regular periodic phases of mitotic activity and rest (the hair cycle). The eventual result of the insult depends, therefore, not only on the force of the drug but also on the phase or subphase in which the insult finds the 
Fig. 4. Left upper lip, 62 days after the first infusion and 10 days after the fifth. There is an evident rarefaction of the hairs, numerous black and white dots (cadaverized hairs) are present that do not seem having grown up. Some of them, instead, have originated short hairs (black circles). Numerous white hairs that have grown up attaining some length are visible. They all are dystrophic hairs (Fig. 3).

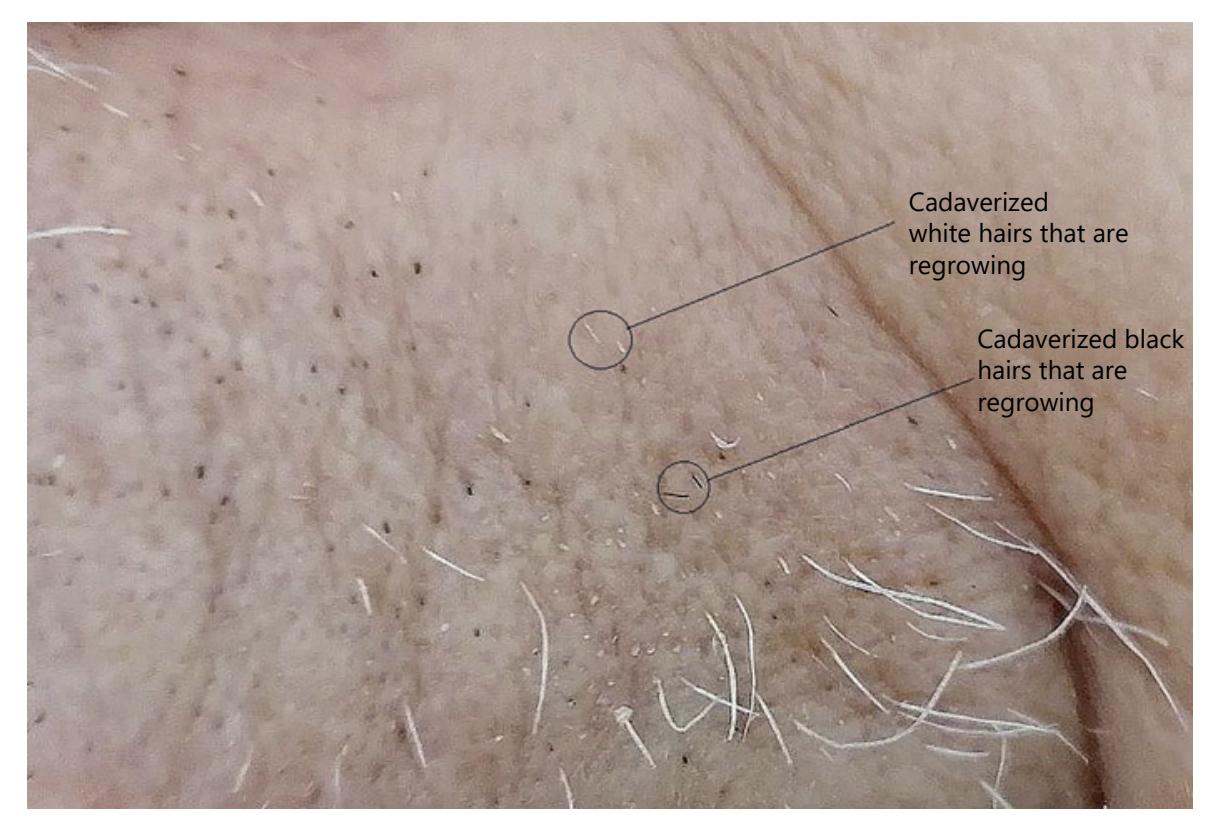

follicle, being ineffective, whatever the potency of the drug, in the phases that are mitotically silent. In brief, 2 additional pathogenetic factors should be taken into account when considering the effect of any antimitotic agent on the hair: the phase of the hair cycle in which the insult finds the hair follicle and the coexistence of factors that modify the normal length of the cycle phases.

Back to the "several factors" mentioned earlier, the information available in the literature concerning the rates of the hair growth is regrettably scanty. Vogt et al. [16] report that scalp and beard hairs grow at roughly the same rate $(0.35$ and $0.38 \mathrm{~mm} /$ day, respectively), while eyebrows grow more slowly $(0.16 \mathrm{~mm} /$ day $)$. Racial differences have been described [17] and, indeed, in Japanese, the growth rate on the vertex $(0.44 \mathrm{~mm} /$ day $)$ is higher than on the temporal area $(0.39 \mathrm{~mm} /$ day $)$ and, in general, than in Caucasians. In Japanese, beard hairs, instead, would not grow as fast $(0.27 \mathrm{~mm} /$ day $)$ as scalp hairs [18]. Overall, though, scalp and beard hairs display a high rate of growth and, thus, are extremely susceptible to any cytostatic insult. Incidentally, beard hairs undergo seasonal variations, growing faster in July and reaching a minimum in January and February [19]. How this may influence the effect of chemotherapy on hair should be studied.

As for the effect of age on the cell division rates, Tomasetti et al. [20] report significantly slowed division rates with age in the human self-renewing tissues they studied. If this were true even for the hair, therefore, in elderly people undergoing chemotherapy alopecia should be less frequent or even less extensive than in youngsters. Actually, age is reported to be associated with neither the severity nor the frequency rates of chemotherapy alopecia [21], but the regrowth of eyebrows, eyelash, and body hairs is accelerated in the premenopausal patients [21]. In apparent contrast to this view, white beard hairs, which are obviously more common in aged people, grow more than twice as fast $(1.12 \mathrm{~mm} /$ day $)$ as pigmented hairs $(0.47$ $\mathrm{mm}$ /day) [22] and, therefore, are supposedly more susceptible to antimitotic agents. In the direct experience of one of us, in fact, the beard thinned out after docetaxel (50 $\mathrm{mg} / \mathrm{m}^{2}$ every 15 days) along with scalp hairs, and both its black and white hairs exhibited the highest degree of dystrophy ("cadaverized" hairs) (Fig. 2a, b). Yet, most white hairs continued to grow during the treatment though exhibiting, once plucked, some dystrophy (Fig. 3), somehow confirming the laypeople belief that white hairs are tougher than pigmented hairs. In fact, white (gray) hairs have a broader shaft than pigmented hairs [23], and dystrophy may find it difficult to affect their whole shaft, preventing its full break and hair shedding. This may explain why, despite the prevalence of white hairs, the black cadaverized hairs predominate (Fig. 2b, 4).

Last, AGA is a condition that modifies the length of the various phases of the hair cycle. AGA, however, has seldom been considered in chemotherapy-induced hair loss, and even the comprehensive essay of Rugo and Lacouture cites it only incidentally [2]. Yet, AGA is a very peculiar affection being more common than normality, no matter 
the gender, at least among Caucasians, to the point that a renowned biologist like William Montagna wrote, perhaps a little too boldly, "normally, all human beings become progressively balder" [24].

In both genders, AGA is characterized not only by the progressive reduction of the number of terminal hairs but also and especially by the simultaneous increase in the number of vellus hairs, the so-called miniaturization. Hair miniaturization is the currently accepted pathogenetic mechanism of AGA. Actually, it clarifies why, in the balding subject, the hair shafts become progressively thinner but hardly explains why they also rarefy [25]. What matters here, however, is that the normal rhythm of the hair cycles is, in balding subjects, accelerated, with a relative prevalence of catagen-telogen phases. This detail is not futile but deeply influences the outcome of chemotherapy on scalp hairs.

The phase of the cycle in which the insult finds the follicle is a crucial point. If the follicle is in a subphase with the topmost mitotic activity (anagen subphases I-IV), a quantity of mitoses would be blocked and the hair would be shed as a dystrophic hair (AE). Conversely, if the injured follicle is approaching the end of the anagen phase (anagen phases V and VI), in which the mitotic rate is already spontaneously declining, what occurs is the simple acceleration of its normal progress to telogen. As a mitotically inactive phase, telogen will, then, become a sanctuary for the insulted hair to take refuge for 3 months (the normal length of telogen) before being normally shed (telogen effluvium [TE]).

When AGA coexists, the ratio between the length of anagen and that of telogen becomes a critical factor for the quality of the hair response to the insult. If this ratio is low, as in AGA, in which the anagen length is abbreviated, the chances for the insult to find keratinocytes with a high mitotic rate are reduced. As AGA is very common among Caucasians, therefore, the anagen/telogen length ratio is, in most patients, but especially in men, particularly low, with the prevalence of the telogen duration. The type of shedding would be, therefore, not only an early $\mathrm{AE}$ but also a late TE, which occurs only when the telogen phase is terminated, that is, 3 months later.

In brief, the same antimitotic insult may cause $\mathrm{AE}$ or TE, irrespective of its quality. Even a combination of both effluvia is possible.

Again, in biology, nothing is simple. When a hair dystrophy occurs, hairs are supposed to shed immediately and profusely. Actually, oncologists maintain that hairs start shedding 2 or 3 weeks after the first course of chemotherapy [2], an observation also suggested by trichos-

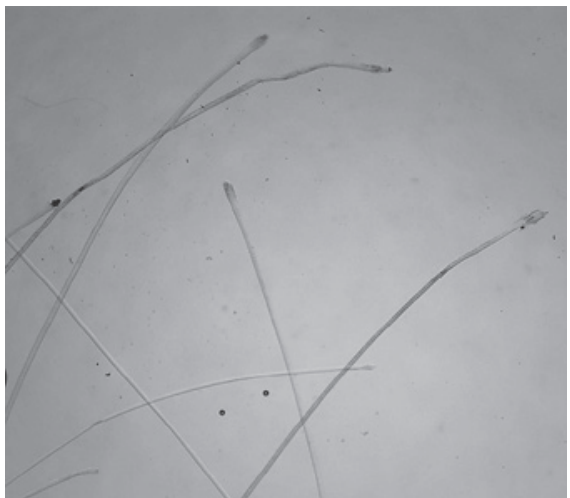

Fig. 5. A tuft of scalp hairs 108 days after the first infusion and 39 after the last one. All hairs have been converted to telogen hairs, although some of them have an irregular shape.

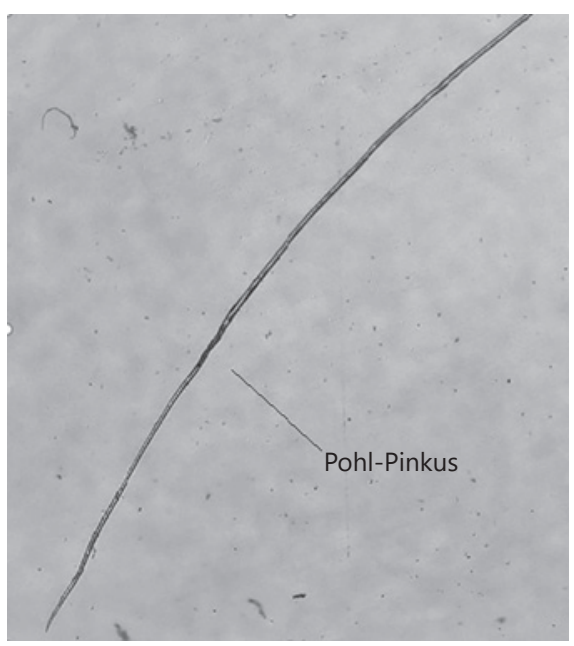

Fig. 6. One of the white beard hairs, which survived the docetaxel infusions, has grown up further ( 3 months after the first infusion and 4 days before the last one), though severely dystrophic. Note the Pohl-Pinkus constriction (arrow) that probably corresponds to one of the infusions.

copy [26]. According to Yun et al. [11], instead, hairs start to shed 7-10 days after the initiation of chemotherapy and, in the mentioned personal experience of one of us, began shedding just $4-5$ days after the first docetaxel infusion, continuing to do so for at least 3 weeks. Hair loss became gradually less copious even in constancy of treatment and, about 3 months later, converted to TE (Fig. 5).

Such difference in times is difficult to explain as there are no apparent biological reasons for any delay in between the initiation of the antimitotic treatment and its eventual result on the hair shaft (dystrophy). Being dystrophic, however, does not mean that the affected hairs shed immediately. A number of dystrophic hairs, includ- 
ing even some of the "cadaverized" ones, survive the first antimitotic attack and continue to grow (Fig. 2, 4) even during the treatment, though less than expected, confirming that the damaged bulb structures are rapidly restored [2]. Regrettably, an equivalent process might occur to the cancer cells as well, probably depending on the specificity of the drug. It may be, in any case, that people who do not complain of hair loss after chemotherapy have, in fact, undergone dystrophy but not such as to cause an immediate shedding.

Another possible, and not mutually exclusive, explanation is given by the TE type of shedding. In such a case, hairs start falling about 3 months after the insult, that is, the time that the hair takes to stay in the telogen phase before shedding spontaneously. This time lag is usually ignored by patients. Late hair shedding may be overlooked and its pathogenetic connection with the chemotherapy disregarded. The immediate $\mathrm{AE}$ is a dramatic event and cannot be ignored, while the late TE may go unnoticed, especially if affecting an already impoverished scalp.

In conclusion, all patients undergoing chemotherapy are likely to display hair dystrophy of, mainly, scalp and beard hairs as they are characterized by a high mitotic rate. Such dystrophy, however, does not always result in an immediate hair shedding. Some hairs, in fact, though dystrophic, not only do not shed but are rapidly restored and continue to grow for weeks, dodging the following drug administrations (Fig. 6). Whether an equivalent process occurs to cancer cells as well remains to be established. The eventual outcome rests, of course, on the force of the drug but also on the presence of AGA, in which a late TE is more probable. It is likely that in women, in whom AGA is less severe, the hair loss is more intense and, mostly, of the AE type, while in men, due to the higher severity of AGA, the AE is less copious and full hairlessness less common and probably belated.

\section{Acknowledgements}

We wish to thank Valentina Rebora for taking the picture of Figure 5.

\section{Conflict of Interest Statement}

The authors have no conflicts of interest to declare and had no funding sources.

\section{Author Contributions}

Both authors contributed equally to the research.

\section{References}

1 Huang W-Y, Hong J-B, Chang M, Wang S-Y, Lai S-F, Chein H-F, et al. Lower proximal cup and outer root sheath cells regenerate hair bulbs during anagen hair follicle repair after chemotherapeutic injury. Exp Dermatol. 2020 Aug 11.

2 Rugo HS, Lacouture ME. Alopecia related to systemic cancer therapy. Available from: https://www.uptodate.com/contents.

3 Fairlamb DJ. Hair changes following cytotoxic drug induced alopecia. Postgrad Med J. 1988;64(757):907.

4 Kluger N, Jacot W, Frouin E, Rigau V, Poujol S, Dereure O, et al. Permanent scalp alopecia related to breast cancer chemotherapy by sequential fluorouracil/epirubicin/cyclophosphamide (FEC) and docetaxel: a prospective study of 20 patients. Ann Oncol. 2012;23(11): 2879-84.

5 Núñez-Torres R, Martín M, García-Sáenz JA, Rodrigo-Faus M, Del Monte-Millán $\mathrm{M}$, Tejera-Pérez $\mathrm{H}$, et al. Association between $\mathrm{ABCB} 1$ genetic variants and persistent chemotherapy-induced alopecia in women with breast cancer. JAMA Dermatol. 2020;5: 201867.
6 Tosti A, Palamaras I, Miteva M, Misciali C. Docetaxel and permanent alopecia. J Am Acad Dermatol. 2013;68(5):e151.

7 Tallon B, Blanchard E, Goldberg LJ. Permanent chemotherapy-induced alopecia: case report and review of the literature. J Am Acad Dermatol. 2010;63(2):333-6.

8 Rebora A, Semino MT, Guarrera M. Trichodynia. Dermatology. 1996;192(3):292-3.

9 Kanti V, Nuwayhid R, Lindner J, Hillmann K, Bangemann N, Kleine-Tebbe A, et al. Evaluation of trichodynia (hair pain) during chemotherapy or tamoxifen treatment in breast cancer patients. J Eur Acad Dermatol Venereol. 2016;30(1):112-8.

10 Sengelov L, Kamby C, Lund B, Engelholm SA. Docetaxel and cisplatin in metastatic urothelial cancer: a phase II study. J Clin Oncol. 1998; 16:3392-7.

11 Yun SJ, Kim SJ. Hair loss pattern due to chemotherapy-induced anagen effluvium: a cross-sectional observation. Dermatology. 2007;215(1):36-40.
12 Sibaud V, Lebœuf NR, Roche H, Belum VR Gladieff L, Deslandres M, et al. Dermatological adverse events with taxane chemotherapy. Eur J Dermatol. 2016;26(5):427-43.

13 Weinstein GD, Mooney KM. Cell proliferation kinetics in the human hair root. J Invest Dermatol. 1980;74(1):43-6.

14 Batchelor D. Hair and cancer chemotherapy: consequences and nursing care-a literature study. Eur J Cancer Care. 2001;10(3):147-63.

15 Dunnill CJ, Al-Tameemi W, Collett A, Haslam IS, Georgopoulos NT. A clinical and biological guide for understanding chemotherapy-induced alopecia and its prevention. Oncologist. 2018;23(1):84-96.

16 Vogt A, McElvee MJ, Blume-Peytawi U. Biology of hair follicle. In: Blume-Peytawi U, Tosti A, editors. Hair growth and disorders. Berlin: Springer; 2008. p. 1-22.

17 Myers RJ, Hamilton JB. Regeneration and rate of growth of hairs in Man. Ann N Y Acad Sci. 1951;53(3):562-8.

18 Saitoh M, Makoto U, Sakamoto M. Rate of hair growth. In: Montagna W, Dobson RL, editors. Hair growth. Oxford: Pergamon Press; 1967. p. 183-203. 
19 Randall VA, Ebling FJ. Seasonal changes in human hair growth. Br J Dermatol. 1991; 124(2):146-51.

20 Tomasetti C, Poling J, Roberts NJ, London NR, Pittman ME, Haffner MC, et al. Cell division rates decrease with age, providing a potential explanation for the age-dependent deceleration in cancer incidence. Proc Natl Acad Sci U S A. 2019;116(41):20482-8.

21 Fujii T, Ichiba K, Honda C, Tokuda S, Nakazawa Y, Ogino M, et al. Prospective observational study of chemotherapy-induced alopecia after sequential FEC+ taxane and the effects of age in breast cancer patients. Breast Cancer. 2020 Sep 17.
22 Maurer M, Rietzler M, Burghardt R, Siebenhaar F. The male beard hair and facial skin challenges for shaving. Int J Cosmet Sci. 2016; 38 Suppl 1(Suppl 1):3-9.

23 Hamilton JB, Terada H, Mestler GE, Tirman WI. Coarse sternal hairs, a male secondary sex character that can be measured quantitatively: the influence of sex, age and genetic factors. In: Montagna W, Dobson RL, editors. Hair growth. Oxford: Pergamon Press; 1967. p. 139.
24 Montagna W. Baldness: a disease? JAMWA. 1973;29:447-58.

25 Guarrera M, Rebora A. The higher number and longer duration of kenogen hairs are the main cause of the hair rarefaction in androgenetic alopecia. Skin Appendage Disord. 2019; 5(3):152-4.

26 Rossi A, Caterina Fortuna M, Caro G, Cardone M, Garelli V, Grassi S, et al. Monitoring chemotherapy-induced alopecia with trichoscopy. J Cosmet Dermatol. 2019;18(2):575-80. 